\title{
SKA - EoR correlations and cross-correlations: kSZ, radio galaxies, and NIR background
}

\author{
Vibor Jelić $^{1,2 *}$, Benedetta Ciardi $^{3}$, Elizabeth Fernandez ${ }^{1}$, Hiroyuki Tashiro ${ }^{4}$, Dijana \\ Vrbanec $^{3}$ \\ ${ }^{1}$ Kapteyn Astronomical Institute, University of Groningen, PO Box 800, 9700 AV Groningen, the \\ Netherlands; \\ ${ }^{2}$ ASTRON - the Netherlands Institute for Radio Astronomy, PO Box 2, 7990 AA Dwingeloo, the \\ Netherlands; \\ ${ }^{3}$ Max-Planck Institute for Astrophysics, Karl-Schwarzschild-Strasse 1, D-85748 Garching bei \\ München, Germany; \\ ${ }^{4}$ Department of Physics and Astrophysics, Nagoya University, Furocho, Chikusaku, Nagoya, \\ 464-8602 Japan \\ E-mail: vjeliceastro.rug.nl
}

\begin{abstract}
The Universe's Cosmic Dawn (CD) and Epoch of Reionization (EoR) can be studied using a number of observational probes that provide complementary or corroborating information. Each of these probes suffers from its own systematic and statistical uncertainties. It is therefore useful to consider the mutual information that these data sets contain. In this paper, we discuss a potential of cross-correlations between the SKA cosmological $21 \mathrm{~cm}$ data with: (i) the kinetic SunyaevZel'dovich (kSZ) effect in the CMB data; (ii) the galaxy surveys; and (iii) near infrared (NIR) backgrounds.
\end{abstract}

Advancing Astrophysics with the Square Kilometre Array

June 8-13, 2014

Giardini Naxos, Italy

\footnotetext{
* Speaker.
} 


\section{Introduction}

Experiments designed to measure the redshifted $21 \mathrm{~cm}$ line from the Cosmic Dawn (CD) and Epoch of Reionization (EoR) are challenged by the strong astrophysical foreground contamination, ionospheric distortions, radio frequency interference and complex instrumental response. In order to reliably detect the cosmological signal from the observed data, it is essential to understand in detail all aspects of the experiment. For example, the cosmological signal has some characteristics which differentiates it from the foregrounds and noise. By use of proper statistics, it is possible to remove these components to extract signatures of reionization (see Chapman et al. 2014).

To alleviate some of the problems associated with the observations of the weak cosmological signal, several cross-correlation analyses with observations in other frequency windows have been proposed. In doing this, the noise/systematics in two observations of different frequencies and strategies may cancel out. Even if SKA may have a high enough sensitivity not to need crosscorrelation techniques in order to detect the signal, cross-correlating with other probes will improve our understanding of the process of reionization (for an overview Mellema et al. 2013).

In this paper we present the potential of cross-correlation studies between the SKA cosmological $21 \mathrm{~cm}$ data, which reflects the neutral hydrogen content of the Universe as a function of redshift, with: (i) the kinetic Sunyaev-Zel'dovich (kSZ) effect in the Cosmic Microwave Background (CMB) data, produced by the scattering of CMB photons off free electrons produced during the reionization process (Sec. 2); (ii) galaxy surveys (Sec. 3); and (iii) the near infrared (NIR) backgrounds that reflect the primordial star formation (Sec. 4).

Throughout this study we consider three SKA configurations: early science SKA1-LOW, SKA1-LOW and SKA2-LOW. The SKA1-LOW (Dewdney et al. 2013) is our standard SKA configuration for the noise simulations, using the OSKAR simulator ${ }^{1}$. We assume full correlation between all 866 core stations of SKA1-LOW, where the maximum baseline length is $5.29 \mathrm{~km}$. Baseline coordinates are generated for a 12-hour synthesis observation, with a 5-minute sampling interval. The noise is then re-normalised to reflect 1000 hours of integration. For early science SKA1-LOW we assume that the sensitivity is halved. For SKA2-LOW we assume that the sensitivity is quadrupled.

\section{The Cross-Correlation with the $\mathrm{kSZ}$}

One of the leading sources of secondary anisotropies in the CMB is due to the scattering of CMB photons off free electrons (Zeldovich \& Sunyaev 1969). The effect of anisotropies when induced by thermal motions of free electrons is called the thermal Sunyaev-Zel'dovich effect (tSZ) and when due to bulk motion of free electrons is called the kinetic Sunyaev-Zel'dovich effect (kSZ). The latter is far more dominant during reionization (for a review of secondary CMB anisotropies see, e.g. Aghanim et al. 2008).

The kSZ effect from a homogeneously ionized medium, i.e., with ionized fraction only as a function of redshift, has been studied both analytically and numerically by a number of authors; the linear regime of this effect was first calculated by Sunyaev \& Zeldovich (1970) and subsequently revisited by Ostriker \& Vishniac (1986) and Vishniac (1987) - hence also referred to as

\footnotetext{
${ }^{1}$ http://www.oerc.ox.ac.uk/ ska/oskar
} 


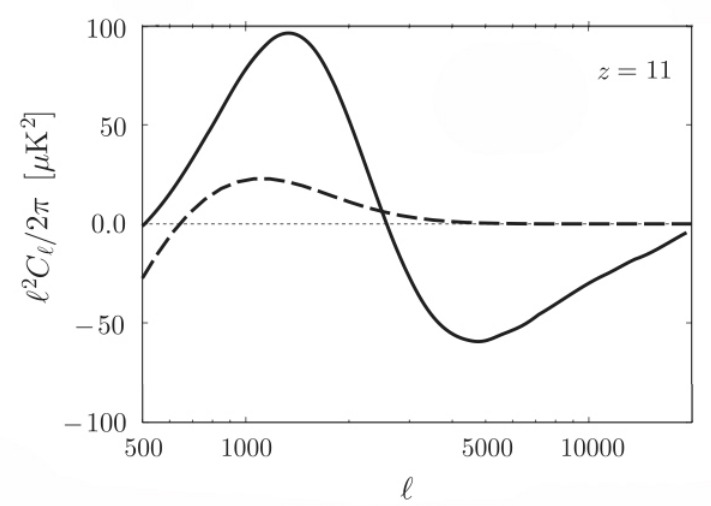

Figure 1: An example of the cross-power spectrum of the $\mathrm{kSZ}$ and the cosmological $21 \mathrm{~cm}$ signal at $z=11$. The solid line is for a 'patchy' reionization history, while the dashed line is for a 'homogenous' history. (Tashiro et al. 2011)

the Ostriker-Vishniac (OV) effect. In recent years various groups have calculated this effect in its non-linear regime using semi-analytical models and numerical simulations (Gnedin \& Jaffe 2001; Santos et al. 2003; Zhang et al. 2004). These studies show that the contributions from non-linear effects are only important at small angular scales $(l>1000)$, while the OV effect dominates at larger angular scales.

The kSZ effect from patchy reionization was first estimated using simplified semi-analytical models by Santos et al. (2003), who concluded that it dominates over that of a homogeneously ionized medium. More detailed modeling of the effect of patchy reionization were subsequently performed using numerical simulations (Salvaterra et al. 2005; Iliev et al. 2007) and semi-analytical models (McQuinn et al. 2005; Zahn et al. 2005; Mesinger et al. 2012). Doré et al. (2007) used numerical simulations to derive the expected CMB polarization signals due to EoR patchiness. The CMB bolometric arrays Atacama Cosmology Telescope (ACT, Fowler et al. 2010) and South Pole Telescope (SPT, Shirokoff et al. 2011) are currently being used to measure the CMB anisotropies at the scales relevant to reionization $(3000<\ell<8000)$. The SPT results are starting to put limits on the duration of reionization (Zahn et al. 2012).

Cross-correlation between the cosmological $21 \mathrm{~cm}$ signal, as measured with SKA, and the secondary CMB anisotropies provide a potentially useful statistic. The cross-correlation has the advantage that the measured statistic is less sensitive to contaminants such as the foregrounds, systematics and noise in comparison to "auto-correlation" studies.

Analytical cross-correlation studies between the CMB temperature anisotropies and the EoR signal on large scales $(l \sim 100)$ were carried out by Alvarez et al. (2006); Adshead \& Furlanetto (2008); Lee (2009) and on small scales $(l>1000)$ by Cooray (2004); Salvaterra et al. (2005); Slosar et al. (2007); Tashiro et al. (2008, 2010, 2011). Cross-correlation between the E- and B-modes of CMB polarization with the redshifted $21 \mathrm{~cm}$ signal was done by Tashiro et al. (2008); Dvorkin et al. (2009). Numerical studies of the cross-correlation were carried out by Salvaterra et al. (2005); Jelić et al. (2010).

These studies showed that the kSZ and the redshifted $21 \mathrm{~cm}$ signal: (i) anti-correlate on the scales corresponding to the typical size of ionized bubbles; and (ii) correlate on the larger scales, 

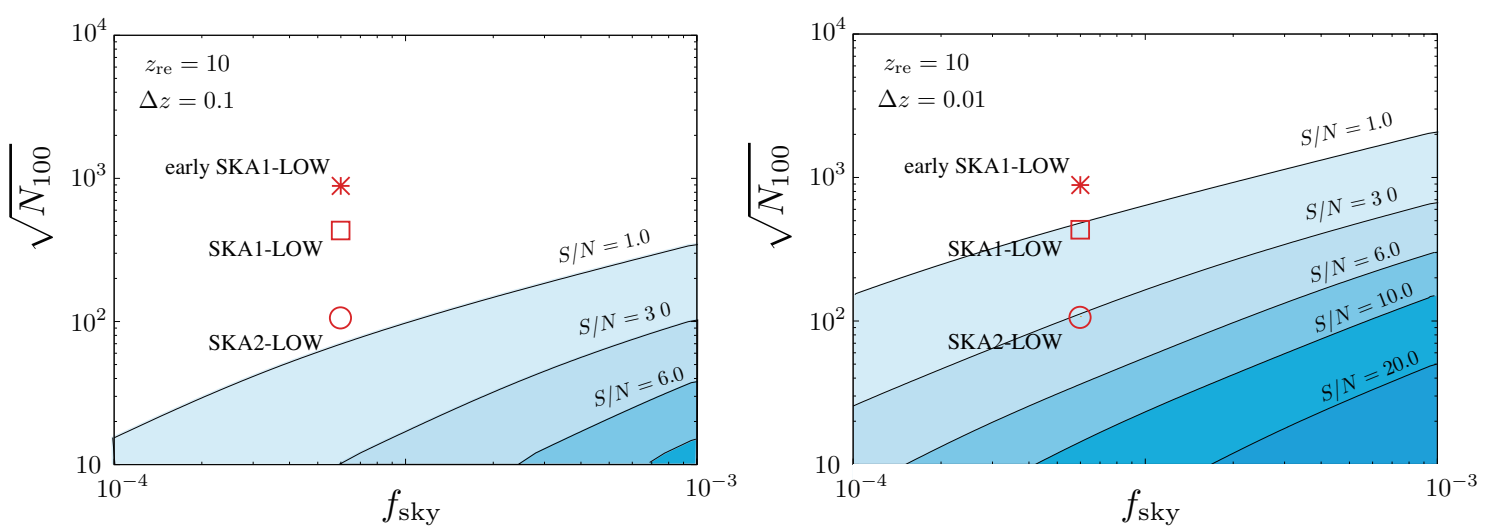

Figure 2: $\mathrm{S} / \mathrm{N}$ of the $21 \mathrm{~cm}$ cross-correlation with the $\mathrm{kSZ}$ for two different reionization models, defined with the redshift at which the ionized fraction equals $0.5\left(z_{\text {re }}\right)$ and the reionization duration $(\Delta z)$. The $\mathrm{S} / \mathrm{N}$ is given as a function of the sky fraction and the normalised noise power spectrum. We assume 1000h of integration time with early SKA1-LOW, SKA1-LOW and SKA2-LOW and a field of view of $25 \mathrm{deg}^{2}$. For the CMB data we assume Planck sensitivity. (based on Tashiro et al. 2010)

where the patchiness of the ionization bubbles are averaged out (see Fig. 1). The significance of the anti-correlation signal depends on the reionization scenario (Salvaterra et al. 2005; Jelić et al. 2010; Tashiro et al. 2011).

The cross-correlation signal turns out to be difficult to detect (see Fig. 2). We might be able to make detection only with SKA2-LOW, assuming a very radical reionization duration of $\Delta z=0.01$. If duration of reionization is longer, detection is not possible for all three SKA configurations. However, the kSZ signal induced during the EoR could possibly be detected in the power spectra of the $\mathrm{CMB}$ and used to place some additional constraints on this epoch in the history of our Universe.

\section{The Cross-Correlation with Galaxy Surveys}

Following Lidz et al. (2009), one can define the cross power spectrum between the $21 \mathrm{~cm}$ emission and the galaxies as:

$$
\begin{aligned}
\Delta_{21, \mathrm{gal}}^{2}(k)= & \tilde{\Delta}_{21, \mathrm{gal}}^{2}(k) / \delta T_{b 0} \\
= & x_{\mathrm{HI}}\left[\Delta_{x_{\mathrm{HI}}, \mathrm{gal}}^{2}(k)+\Delta_{\rho, \mathrm{gal}}^{2}(k)\right. \\
& \left.+\Delta_{\rho x_{\mathrm{HI}}, \mathrm{gal}}^{2}(k)\right],
\end{aligned}
$$

where $\delta T_{b 0}$ is the $21 \mathrm{~cm}$ brightness temperature relative to the $\mathrm{CMB}$ for neutral gas at the mean density of the universe, $x_{\mathrm{HI}}$ is the neutral fraction and $\Delta_{a, b}^{2}(k)$ is the dimensionless cross power spectrum between fields $a$ and $b$. In order to construct the cross power spectrum, one therefore requires three fields, the density field $(\rho)$, the neutral hydrogen field $\left(x_{\mathrm{HI}}\right)$, and the galaxy field (gal), which can be obtained via numerical simulations of galaxy formation and the reionization process.

It is found that the $21 \mathrm{~cm}$ emission is initially correlated with galaxies on large scales, anticorrelated on intermediate scales, and uncorrelated on small scales. This picture quickly changes 


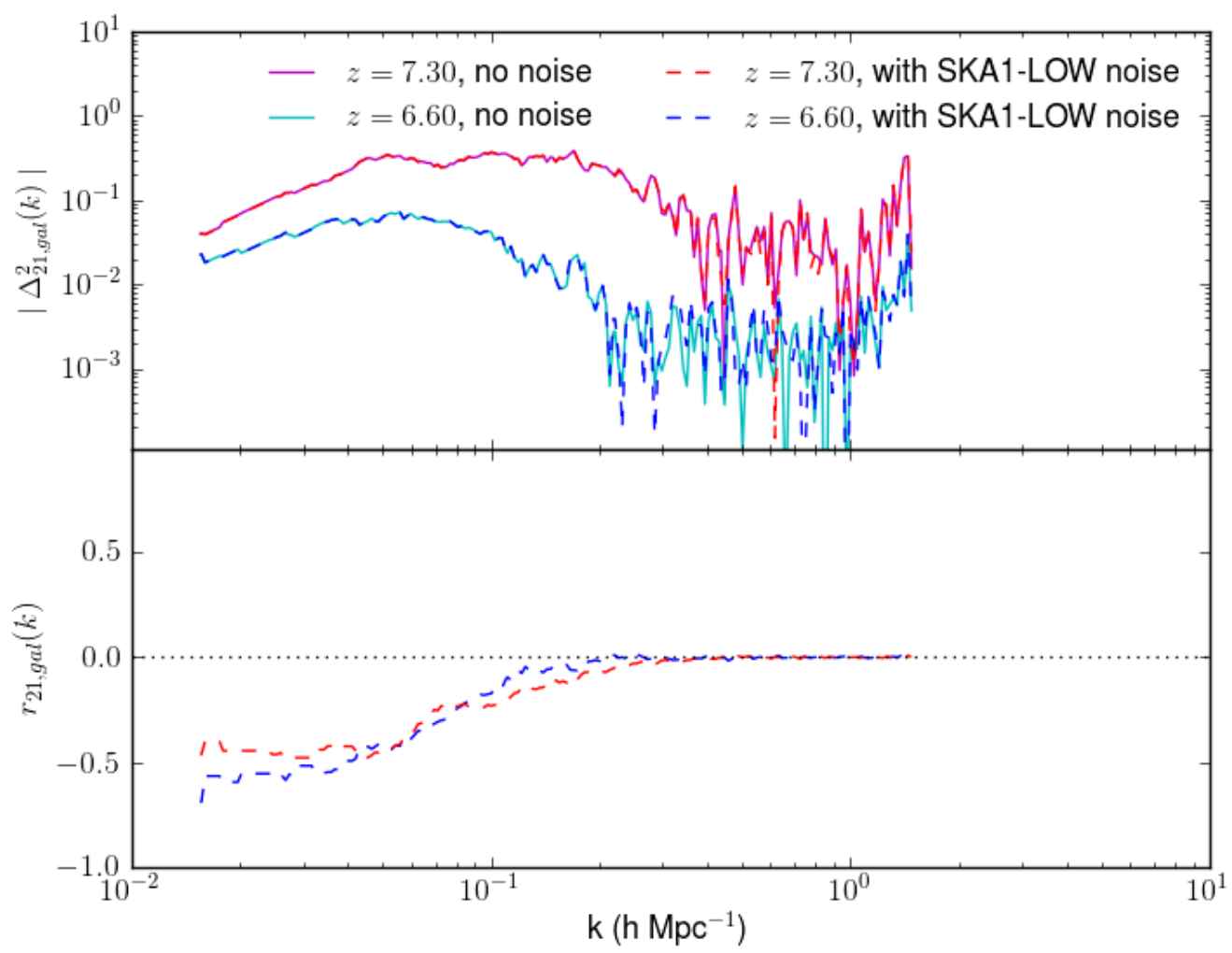

Figure 3: The circularly averaged, unnormalized 2D $21 \mathrm{~cm}$ - galaxy cross power spectrum $\left(\tilde{\Delta}_{21, \mathrm{gal}}^{2}(k)\right.$; and correlation coefficient for two redshifts. In the analysis we followed Wiersma et al. (2013).

as reionization proceeds and the two fields become anti-correlated on large scales (Lidz et al. 2009; Wiersma et al. 2013). These (anti-) correlations can be a powerful tool in indicating the topology of reionization and should form important diagnostic tools for SKA observations.

If the effect of observing and selecting real galaxies is taken into account, the result depends on the observational campaign considered. For example, for a drop-out technique (as in observations of Lyman Break Galaxies), the normalization of the cross power spectrum seems to be the most powerful tool for probing reionization. In particular, it is quite sensitive to the ionized fraction as different reionization histories yield similar cross power spectra for a fixed ionized fraction. When instead a more precise measurement of the galaxy redshifts is available (as in Ly- $\alpha$ Emitters surveys), which provides the three-dimensional position of the galaxy, much more information about the nature of reionization can be extracted, since the shape and the normalization of the cross power spectrum provide useful information. In addition, the observability of the Ly- $\alpha$ line from these galaxies is affected by neutral patches in the IGM and thus Ly- $\alpha$ Emitters surveys are particularly useful for EoR studies (McQuinn et al. 2007; Jensen et al. 2014).

Figure 3 shows the $21 \mathrm{~cm}$ - Ly- $\alpha$ Emitters cross power spectrum (with and without SKA noise) and correlation coefficient for two redshifts. Here the noise assigned to the $21 \mathrm{~cm}$ survey is the one 
of the SKA telescope with SKA1-LOW configuration and noise for 1000 hours of integration time, while the Ly- $\alpha$ Emitters survey has the same characteristics of the one described in Jensen et al. (2014); Ouchi et al. (2010) with the Subaru telescope. As we can see, the $21 \mathrm{~cm}-$ Ly- $\alpha$ Emitters cross power spectrum is not very sensitive to noise. Therefore, early SKA1-LOW and SKA2-LOW will show very similar results. The effect of neutral patches in the IGM on the observability of these Ly- $\alpha$ Emitters is not included here.

\section{The Cross-Correlation with Near Infrared Backgrounds}

Understanding star formation at high redshifts is fundamentally linked to our understanding of reionization history. In order for reionization to occur, a plentiful source of ionizing photons was needed. Stars are a likely candidate to produce these photons. Therefore, any attempt to understand reionization must be paired with an understanding of primordial star formation.

However, current observational constraints suggest that in order for the Universe to be reionized, a plentiful number of small galaxies beneath the detection limit of current surveys are most likely needed. Because they are so faint, it is difficult to obtain information about these galaxies directly. Instead, these galaxies can be observed using indirect means. For example, the cumulative light from these galaxies would be redshifted to the infrared, and therefore, any background in the infrared may provide clues to the nature of these galaxies. Many have suggested that the remnant light from these galaxies is indeed present in the excess emission in the Near Infrared Background (NIRB), and if this is true, looking at the mean intensity and the power spectrum of the NIRB can give information about these early stellar populations (e.g., Santos et al. 2002; Magliocchetti et al. 2003; Salvaterra \& Ferrara 2003; Cooray 2004; Kashlinsky 2005; Fernandez \& Komatsu 2006; Fernandez et al. 2010; Cooray et al. 2012; Fernandez et al. 2012; Kashlinsky et al. 2012; Fernandez \& Zaroubi 2013; Yue et al. 2013).

On the other hand, $21 \mathrm{~cm}$ emission originates from areas of neutral hydrogen, so it corresponds to regions that have not seen plentiful star formation and hence have not yet been ionized. Therefore, we should expect that regions that are bright in $21 \mathrm{~cm}$ background emission should not be bright in the infrared. Conversely, regions that are bright in the infrared have plenty of star formation and therefore should be dim in the $21 \mathrm{~cm}$ background. Because of this, these two observations should be anti-correlated.

In Fernandez et al. (2014), we predict the observational properties of both high redshift galaxies (which would be observable in the infrared) and the neutral regions that have yet to be ionized by galaxies (observable as part of the $21 \mathrm{~cm}$ background). In order to do this, we combined large scale N-body simulations (Iliev et al. 2014), which included radiative transfer, with analytical models of the luminosities of the halos (Fernandez \& Komatsu 2006). The brightness temperature of the $21 \mathrm{~cm}$ background was also computed from the simulation, which depends on the density and neutral fraction of the IGM around the galaxies.

In Fernandez et al. (2014), we then produced simulated sky maps for both the $21 \mathrm{~cm}$ emission and the emission in the infrared. These maps were created by combining the emission models and the simulation output to create luminosity cubes. These cubes were then randomly rotated and stacked along the line of sight to create a three dimensional cuboid. This was projected onto two dimensions to create a simulated sky map in both the radio and the infrared. In Fernandez et al. 

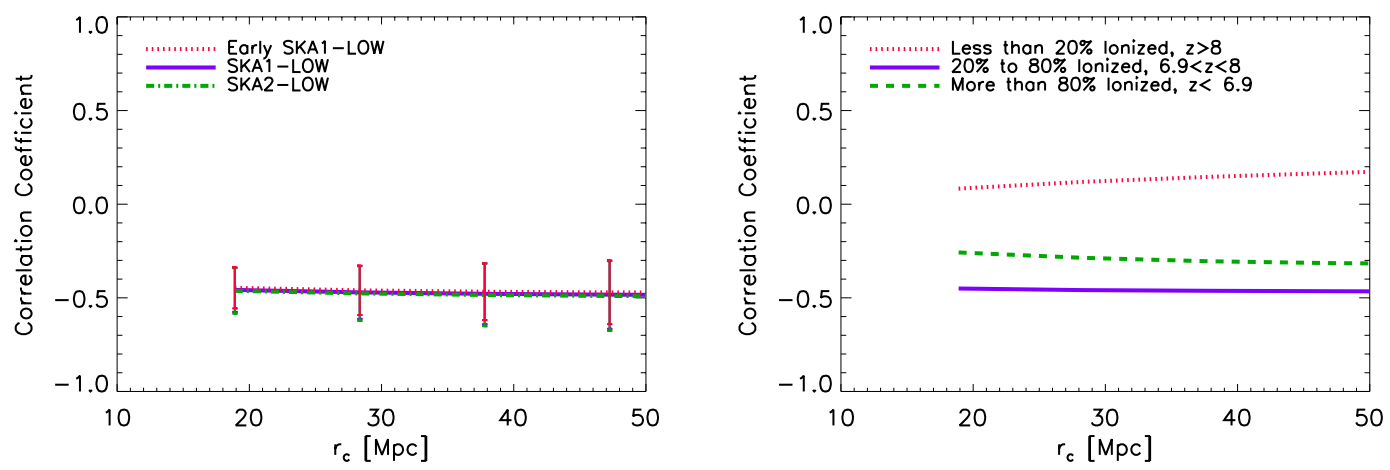

Figure 4: Left: The cross correlation of the entire NIRB against the $21 \mathrm{~cm}$ background, assuming three SKA configurations with $1000 \mathrm{~h}$ of integration time. The results do not depend heavily on noise levels, indicating that errors from unknowns in observation dominate over errors introduced from the noise. Right: The cross correlation at various redshifts: $6.4<z<7,7<z<8$, and $8<z<11$, assuming SKA1-LOW configuration.

(2014), we created sky maps specific for the LOFAR instrument; here, we present maps tailored for three SKA configurations: early science SKA1-LOW, SKA1-LOW and SKA2-LOW (assuming $1000 \mathrm{~h}$ of integration).

For the radio map, since the $21 \mathrm{~cm}$ background is line emission, each frequency corresponds to a different redshift, and observations can be adjusted to correspond to any arbitrary redshift range. On the other hand, since the emission in the infrared has a considerable amount of continuum, it is very difficult to extract redshift information for observations at any given wavelength. Therefore, the map in the infrared is created using the integrated light from a redshift of 6 to 30, or the entire simulation volume.

These two maps in the infrared and in the radio can then be cross-correlated using the Pearson correlation coefficient:

$$
\rho_{21 \mathrm{~cm}, \mathrm{NIRB}}=\frac{\operatorname{cov}\left(\left(\delta T_{\mathrm{b}}\right), I_{N I R B}\right)}{\sigma_{\delta T_{\mathrm{b}}} \sigma_{I_{N I R B}}},
$$

where $I_{N I R B}$ is the intensity in the infrared and $\delta T_{\mathrm{b}}$ is the brightness temperature of the $21 \mathrm{~cm}$ line. The results of this cross-correlation is shown in left panel of Fig. 4, which illustrates the crosscorrelation between the integrated light from $6<z<30$ in the near-infrared against the entire $21 \mathrm{~cm}$ map from $6.4<z<11$, assuming three SKA configurations. Error bars are generated from crosscorrelating the $21 \mathrm{~cm}$ emission with 1000 randomized maps in the infrared. The cross-correlation coefficient shows that these two maps are strongly anti-correlated, corresponding to the fact that the two emission maps are sensitive to emission from mutually exclusive areas. In addition, there is not a significant difference in result between Early SKA1-LOW, SKA1-LOW, and SKA2-LOW, indicating that the errors introduced from the noise is sub-dominant.

In addition, since the $21 \mathrm{~cm}$ background is line emission, we can cross-correlate only specific slices of the $21 \mathrm{~cm}$ background with the entire NIRB. Results of this for SKA1-LOW configuration are shown in the right panel of Fig. 4. The correlation coefficient is computed using radio maps for early $(z>8)$, mid $(6.9<z<8)$ and late $(z<6.9)$ stages of reionization against the entire NIRB. The correlation coefficient is the most negative during mid stages of reionization. At early stages 
of reionization, ionized bubbles around the host galaxies are too small, perhaps smaller than the smoothing length, and therefore no correlation is seen between the two maps. At late stages of reionization, most of the structure in the $21 \mathrm{~cm}$ maps has disappeared.

\section{Conclusions}

In the view of presented results, cross-correlations with other probes will improve our understanding of the process of reionization. The cross-correlation with the galaxy surveys and NIR backgrounds will specifically help in answering the question which types of galaxies are mostly responsible for reionization. There is not a significant difference in results between Early SKA1LOW, SKA1-LOW, and SKA2-LOW, indicating that the errors introduced from the noise is subdominant. Unfortunately, the cross-correlation signal in the case of the CMB turns out to be difficult to detect. There is only a possibility of detection in the case of a very short reionization scenario and using SKA2-LOW.

\section{References}

Adshead, P. J. \& Furlanetto, S. R. 2008, MNRAS, 384, 291

Aghanim, N., Majumdar, S., \& Silk, J. 2008, Reports on Progress in Physics, 71, 066902

Alvarez, M. A., Komatsu, E., Doré, O., \& Shapiro, P. R. 2006, ApJ, 647, 840

Chapman, E., Bonaldi, A., Harker, G., Jelić, V., Abdalla, F., Bernardi, G., Bobin, J., Dulwich, F., Mort, B., Santos, M., \& Starckh, J.-L. 2014, PoS(AASKA14)

Cooray, A. 2004, PhRvD, 70, 063509

Cooray, A., Gong, Y., Smidt, J., \& Santos, M. G. 2012, ApJ, 756, 92

Dewdney, P., Turner, W., Millenaar, R., McCool, R., Lazio, J., \& Cornwell, T. 2013, SKA-TELSKO-DD-001

Doré, O., Holder, G., Alvarez, M., Iliev, I. T., Mellema, G., Pen, U.-L., \& Shapiro, P. R. 2007, PhRvD, 76, 043002

Dvorkin, C., Hu, W., \& Smith, K. M. 2009, ArXiv e-prints

Fernandez, E. R., Iliev, I. T., Komatsu, E., \& Shapiro, P. R. 2012, ApJ, 750, 20

Fernandez, E. R. \& Komatsu, E. 2006, ApJ, 646, 703

Fernandez, E. R., Komatsu, E., Iliev, I. T., \& Shapiro, P. R. 2010, ApJ, 710, 1089

Fernandez, E. R. \& Zaroubi, S. 2013, MNRAS, 433, 2047

Fernandez, E. R., Zaroubi, S., Iliev, I. T., Mellema, G., \& Jelić, V. 2014, MNRAS, 440, 298

Fowler, J. W., Acquaviva, V., Ade, P. A. R., Aguirre, P., Amiri, M., Appel, J. W., Barrientos, L. F., Battistelli, E. S., Bond, J. R., Brown, B., Burger, B., Chervenak, J., Das, S., Devlin, M. J., Dicker, S. R., Doriese, W. B., Dunkley, J., Dünner, R., Essinger-Hileman, T., Fisher, R. P., Hajian, A., Halpern, M., Hasselfield, M., Hernández-Monteagudo, C., Hilton, G. C., Hilton, M., Hincks, A. D., Hlozek, R., Huffenberger, K. M., Hughes, D. H., Hughes, J. P., Infante, L., Irwin, K. D., Jimenez, R., Juin, J. B., Kaul, M., Klein, J., Kosowsky, A., Lau, J. M., Limon, M., Lin, Y.-T., Lupton, R. H., Marriage, T. A., Marsden, D., Martocci, K., Mauskopf, P., Menanteau, F., Moodley, K., Moseley, H., Netterfield, C. B., Niemack, M. D., Nolta, M. R., Page, L. A., Parker, L., Partridge, B., Quintana, H., Reid, B., Sehgal, N., Sievers, J., Spergel, D. N., Staggs, S. T., 
Swetz, D. S., Switzer, E. R., Thornton, R., Trac, H., Tucker, C., Verde, L., Warne, R., Wilson, G., Wollack, E., \& Zhao, Y. 2010, ApJ, 722, 1148

Gnedin, N. Y. \& Jaffe, A. H. 2001, ApJ, 551, 3

Iliev, I. T., Mellema, G., Ahn, K., Shapiro, P. R., Mao, Y., \& Pen, U.-L. 2014, MNRAS, 439, 725 Iliev, I. T., Pen, U.-L., Bond, J. R., Mellema, G., \& Shapiro, P. R. 2007, ApJ, 660, 933

Jelić, V., Zaroubi, S., Aghanim, N., Douspis, M., Koopmans, L. V. E., Langer, M., Mellema, G., Tashiro, H., \& Thomas, R. M. 2010, MNRAS, 402, 2279

Jensen, H., Hayes, M., Iliev, I., Laursen, P., Mellema, G., \& Zackrisson, E. 2014, ArXiv e-prints Kashlinsky, A. 2005, PhysRep, 409, 361

Kashlinsky, A., Arendt, R. G., Ashby, M. L. N., Fazio, G. G., Mather, J., \& Moseley, S. H. 2012, ApJ, 753, 63

Lee, K. 2009, ArXiv e-prints

Lidz, A., Zahn, O., Furlanetto, S. R., McQuinn, M., Hernquist, L., \& Zaldarriaga, M. 2009, ApJ, 690, 252

Magliocchetti, M., Salvaterra, R., \& Ferrara, A. 2003, MNRAS, 342, L25

McQuinn, M., Furlanetto, S. R., Hernquist, L., Zahn, O., \& Zaldarriaga, M. 2005, ApJ, 630, 643

McQuinn, M., Hernquist, L., Zaldarriaga, M., \& Dutta, S. 2007, MNRAS, 381, 75

Mellema, G., Koopmans, L. V. E., Abdalla, F. A., Bernardi, G., Ciardi, B., Daiboo, S., de Bruyn, A. G., Datta, K. K., Falcke, H., Ferrara, A., Iliev, I. T., Iocco, F., Jelić, V., Jensen, H., Joseph, R., Labroupoulos, P., Meiksin, A., Mesinger, A., Offringa, A. R., Pandey, V. N., Pritchard, J. R., Santos, M. G., Schwarz, D. J., Semelin, B., Vedantham, H., Yatawatta, S., \& Zaroubi, S. 2013, Experimental Astronomy, 36, 235

Mesinger, A., McQuinn, M., \& Spergel, D. N. 2012, MNRAS, 422, 1403

Ostriker, J. P. \& Vishniac, E. T. 1986, ApJL, 306, L51

Ouchi, M., Shimasaku, K., Furusawa, H., Saito, T., Yoshida, M., Akiyama, M., Ono, Y., Yamada, T., Ota, K., Kashikawa, N., Iye, M., Kodama, T., Okamura, S., Simpson, C., \& Yoshida, M. 2010, ApJ, 723, 869

Salvaterra, R., Ciardi, B., Ferrara, A., \& Baccigalupi, C. 2005, MNRAS, 360, 1063

Salvaterra, R. \& Ferrara, A. 2003, MNRAS, 339, 973

Santos, M. G., Cooray, A., Haiman, Z., Knox, L., \& Ma, C.-P. 2003, ApJ, 598, 756

Santos, M. R., Bromm, V., \& Kamionkowski, M. 2002, MNRAS, 336, 1082

Shirokoff, E., Reichardt, C. L., Shaw, L., Millea, M., Ade, P. A. R., Aird, K. A., Benson, B. A., Bleem, L. E., Carlstrom, J. E., Chang, C. L., Cho, H. M., Crawford, T. M., Crites, A. T., de Haan, T., Dobbs, M. A., Dudley, J., George, E. M., Halverson, N. W., Holder, G. P., Holzapfel, W. L., Hrubes, J. D., Joy, M., Keisler, R., Knox, L., Lee, A. T., Leitch, E. M., Lueker, M., Luong-Van, D., McMahon, J. J., Mehl, J., Meyer, S. S., Mohr, J. J., Montroy, T. E., Padin, S., Plagge, T., Pryke, C., Ruhl, J. E., Schaffer, K. K., Spieler, H. G., Staniszewski, Z., Stark, A. A., Story, K., Vanderlinde, K., Vieira, J. D., Williamson, R., \& Zahn, O. 2011, ApJ, 736, 61

Slosar, A., Cooray, A., \& Silk, J. I. 2007, MNRAS, 377, 168

Sunyaev, R. A. \& Zeldovich, Y. B. 1970, Astrophys. Space Sci., 7, 3

Tashiro, H., Aghanim, N., Langer, M., Douspis, M., \& Zaroubi, S. 2008, MNRAS, 389, 469

Tashiro, H., Aghanim, N., Langer, M., Douspis, M., Zaroubi, S., \& Jelic, V. 2010, MNRAS, 402, 2617 
Tashiro, H., Aghanim, N., Langer, M., Douspis, M., Zaroubi, S., \& Jelić, V. 2011, MNRAS, 414, 3424

Vishniac, E. T. 1987, ApJ, 322, 597

Wiersma, R. P. C., Ciardi, B., Thomas, R. M., Harker, G. J. A., Zaroubi, S., Bernardi, G., Brentjens, M., de Bruyn, A. G., Daiboo, S., Jelic, V., Kazemi, S., Koopmans, L. V. E., Labropoulos, P., Martinez, O., Mellema, G., Offringa, A., Pandey, V. N., Schaye, J., Veligatla, V., Vedantham, H., \& Yatawatta, S. 2013, MNRAS, 432, 2615

Yue, B., Ferrara, A., Salvaterra, R., \& Chen, X. 2013, MNRAS, 431, 383

Zahn, O., Reichardt, C. L., Shaw, L., Lidz, A., Aird, K. A., Benson, B. A., Bleem, L. E., Carlstrom, J. E., Chang, C. L., Cho, H. M., Crawford, T. M., Crites, A. T., de Haan, T., Dobbs, M. A., Doré, O., Dudley, J., George, E. M., Halverson, N. W., Holder, G. P., Holzapfel, W. L., Hoover, S., Hou, Z., Hrubes, J. D., Joy, M., Keisler, R., Knox, L., Lee, A. T., Leitch, E. M., Lueker, M., Luong-Van, D., McMahon, J. J., Mehl, J., Meyer, S. S., Millea, M., Mohr, J. J., Montroy, T. E., Natoli, T., Padin, S., Plagge, T., Pryke, C., Ruhl, J. E., Schaffer, K. K., Shirokoff, E., Spieler, H. G., Staniszewski, Z., Stark, A. A., Story, K., van Engelen, A., Vanderlinde, K., Vieira, J. D., \& Williamson, R. 2012, ApJ, 756, 65

Zahn, O., Zaldarriaga, M., Hernquist, L., \& McQuinn, M. 2005, ApJ, 630, 657

Zeldovich, Y. B. \& Sunyaev, R. A. 1969, Astrophys. Space Sci., 4, 301

Zhang, P., Pen, U.-L., \& Trac, H. 2004, MNRAS, 347, 1224 